Open Access

\title{
Erratum: Identification and characterization of nuclear and nucleolar localization signals in 58-kDa microspherule protein (MSP58)
}

Chuan-Pin Yang ${ }^{1}$, Chi-Wu Chiang ${ }^{3,4}$, Chang-Han Chen ${ }^{10,9}$, Yi-Chao Lee ${ }^{7,8}$, Mei-Hsiang Wu' ${ }^{1}$ Yi-Huan Tsou ${ }^{2}$, Yu-San Yang ${ }^{3}$, Wen-Chang Chang ${ }^{2,4,6,7}$ and Ding-Yen Lin ${ }^{1,2,4,5^{*}}$

\section{Erratum}

Due to a publisher error in the original version of this article [1], ' $8 \mathrm{~A}$ ' was superimposed on the 10A - EGFP image in Fig. 2C. This error has now been corrected in the original.

\begin{abstract}
Author details
'Institute of Bioinformatics and Biosignal Transduction, College of Bioscience and Biotechnology, National Cheng Kung University, Tainan 70101, Taiwan, ROC. ${ }^{2}$ Department of Pharmacology, College of Medicine, National Cheng Kung University, Tainan 70101, Taiwan, ROC. ${ }^{3}$ Institute of Molecular Medicine, College of Medicine, National Cheng Kung University, Tainan 70101, Taiwan, ROC. ${ }^{4}$ Infectious Diseases and Signaling Research Center, National Cheng Kung University, Tainan 70101, Taiwan, ROC. ${ }^{5}$ Institute for Cancer Biology and Drug Discovery, College of Medical Science and Technology, Taipei Medical University, Taipei 11031, Taiwan, ROC. ${ }^{6}$ Graduate Institute of Medical Sciences, College of Medicine, Taipei Medical University, Taipei 11031, Taiwan, ROC. ${ }^{7}$ Center for Neurotrauma and Neuroregeneration, Taipei Medical University, Taipei 11031, Taiwan, ROC. ${ }^{8}$ Program for Neural Regenerative Medicine, College of Medical Science and Technology, Taipei Medical University, Taipei 11031, Taiwan, ROC. ${ }^{9}$ Center for Translational Research in Biomedical Sciences, Kaohsiung Chang Gung Memorial Hospital, Kaohsiung 83301, Taiwan, ROC. ${ }^{10}$ Department of Applied Chemistry, National Chi Nan University, Puli, Nantou 54561, Taiwan, ROC.
\end{abstract}

Received: 11 June 2015 Accepted: 11 June 2015

Published online: 24 July 2015

\section{Reference}

1. Yang CP, Chiang CW, Chen CH, Lee YC, Wu MH, Tsou YH, et al.

Identification and characterization of nuclear and nucleolar localization signals in 58-kDa microspherule protein (MSP58). J Biomed Sci. 2015;22:33.

\footnotetext{
* Correspondence: lindy@mail.ncku.edu.tw

${ }^{1}$ Institute of Bioinformatics and Biosignal Transduction, College of Bioscience and Biotechnology, National Cheng Kung University, Tainan 70101, Taiwan, ROC

${ }^{2}$ Department of Pharmacology, College of Medicine, National Cheng Kung University, Tainan 70101, Taiwan, ROC

Full list of author information is available at the end of the article
} 


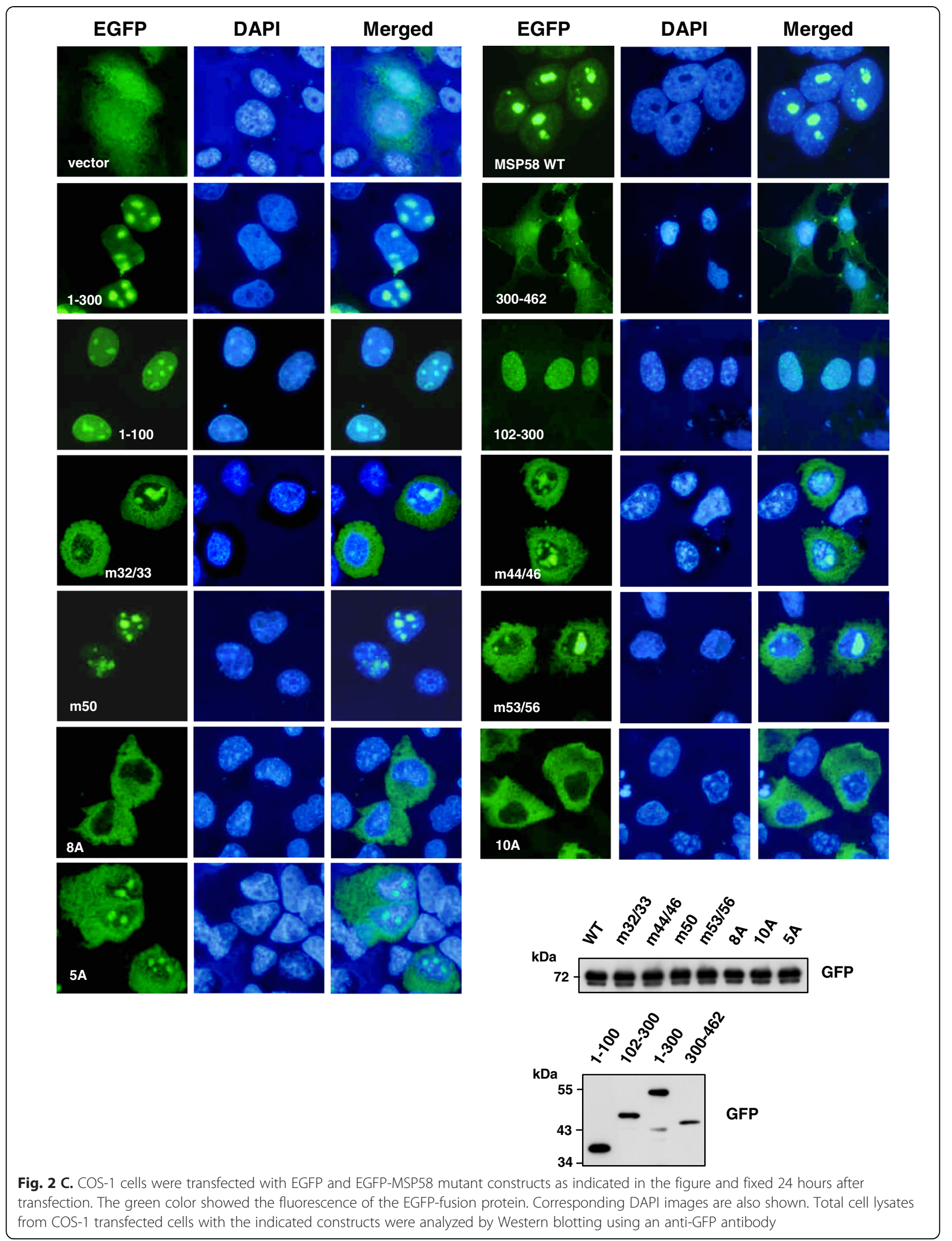

